\title{
Novas ocorrências de desmídias filamentosas (Desmidiaceae, Zygnematophyceae) para o estado da Bahia, Brasil
}

\author{
Ivania Batista de Oliveira ${ }^{1,3}$, Carlos Eduardo de Mattos Bicudo ${ }^{2}$ e Carlos Wallace do Nascimento Moura ${ }^{1}$
}

Recebido em 5/01/2009. Aceito 19/10/2010

RESUMO - (Novas ocorrências de desmídias filamentosas (Desmidiaceae, Zygnematophyceae) do estado da Bahia, Brasil). As desmídias de hábito filamentos são constituídas por filamentos de células relativamente curtos, que se dissociam com certa facilidade. O conhecimento destas algas no estado da Bahia é bastante escasso, até o momento, estando restrito a 13 táxons. O presente trabalho teve por objetivo realizar o inventário taxonômico dos gêneros filamentosos da família Desmidiaceae (Zygnematophyceae), de duas áreas de proteção ambiental, APA Rio Capivara e APA Lagoas de Guarajuba, município de Camaçari, a fim de ampliar o conhecimento destes na Bahia. Foram analisadas 33 amostras coletadas de acordo com os métodos usuais empregados nos estudos de taxonomia de microalgas continentais. Foram identificados 15 táxons distribuídos em sete gêneros (Bambusina Kütz. ex Kütz, Desmidium C.Agardh ex Ralfs, Groenbladia Teiling, Hyalotheca Ehrenb. ex Ralfs, Onychonema Wallich, Spondylosium Bréb. ex Kütz. e Teilingia Bourr.), dos quais nove táxons constituem-se adições à flora de algas continentais da Bahia, aumentando o total de espécies conhecidas para 22.

Palavras-chave: gêneros filamentosos, desmídias, taxonomia

ABSTRACT - (New records of filamentous desmids (Desmidiaceae, Zygnematophyceae) from Bahia state, Brazil). The filamentous desmids are composed of relatively short filaments of cells that dissociate rather easily. These algae are poorly known today in the state of Bahia, being restricted to 13 taxa. This study aimed to do a taxonomic inventory of filamentous genera of the family Desmidiaceae (Zygnematophyceae) from two environmental protection areas, Rio Capivara and Lagoas de Guarajuba, Camaçari municipality, to increase our knowledge of these organisms in Bahia. We analyzed 33 samples collected according to the methods employed in studies of continental microalgae taxonomy. 15 taxa were recorded, distributed in seven genera: Bambusina Kütz. ex Kütz, Desmidium C.Agardh ex Ralfs, Groenbladia Teiling, Hyalotheca Ehrenb. ex Ralfs, Onychonema Wallich, Spondylosium Bréb. ex Kütz. and Teilingia Bourr. Of these, nine are additions to the algal flora of continental Bahia, increasing the total to 22 known species.

Key words: filamentous genera, desmids, taxonomy

\section{Introdução}

A família Desmidiaceae é composta por 36 gêneros, dentre esses, 11 são filamentosos (Bambusina Kütz. ex Kütz, Desmidium C.Agardh ex Ralfs, Groenbladia Teiling, Hyalotheca Ehrenb. ex Ralfs, Onychonema Wallich, Spondylosium Bréb. ex Kütz. and Teilingia Bourr. Phymatodocis Nordst., Shaerozosma Corda ex Ralfs, (Nordst) Racib., Streptonema G.C.Wall. Segundo Bicudo \& Menezes (2006), esse organismos filamentosos compreende aproximadamente 106 táxons.

Os gêneros apresentam filamentos retos ou torcidos em forma de hélices, bainha de mucilagem presente exceto nos gêneros Haploziga e Phymatodocis, com células de formas variadas: cilíndricas, subcilíndricas, globosa, elípticas, às vezes em forma de barrilete, decoradas com estrias, pontos ou espinhos (Brook 1981, Bicudo \& Menezes, 2006).

O conhecimento da biodiversidade das desmídias de hábito filamentoso no estado da Bahia é bastante escasso; até o momento, apenas 13 táxons haviam sido citados e efetivamente publicados para o Estado, os quais encontramse registrados nos trabalhos de Förster (1964), Martins \& Bicudo (1987) e Bicudo \& Martins (1989).

Diante da escassez de conhecimento sobre as desmídias de hábito filamentoso e visando contribuir para o conhecimento da biodiversidade da desmidioflórula do Estado da Bahia, o presente trabalho teve por objetivo realizar o inventário taxonômico dos gêneros filamentosos da família Desmidiaceae de duas Áreas de Proteção Ambiental (APA), a APA Lagoas de
Guarajuba e APA Rio Capivara, ambas situadas no município de Camaçari, litoral norte do estado da Bahia, Brasil.

\section{Material e métodos}

A área de estudo localiza-se no município de Camaçari, Região Metropolitana de Salvador, litoral norte da Bahia e inclui duas áreas de Proteção Ambiental (APA), a APA Lagoas de Guarajuba, com área de 230 ha e a APA Rio Capivara, com área de 1.800 ha (Fig. 1).

O material foi coletado em dois períodos, verão (jan-mar/2007) e inverno (jun-ago/2007), totalizando 33 unidades amostrais, sendo 16 na APA Lagoas de Guarajuba e 17 na APA Rio Capivara. As amostras obtidas de material do fitoplâncton e do perifito foram coletadas de acordo com os métodos usuais empregados nos estudos de taxonomia de microalgas continentais (Bicudo \& Menezes 2006).

As características métricas das populações foram tomadas através de ocular micrometrada e as fotomicrografias obtidas com câmara fotográfica digital ambos acoplados ao microscópio óptico, e as identificações das espécies baseadas em literatura especializada. O sistema de classificação adotado segue Brock (1981).

As amostras foram preservadas em solução de Transeau preparada conforme Bicudo \& Menezes (2006) e tombadas na coleção líquida do Herbário da Universidade Estadual de Feira de Santana (HUEFS).

\section{Resultados e discussão}

Nas Áreas de Proteção Ambiental estudadas, as desmídias filamentosas foram representadas por sete gêneros e 15 táxons. O gênero melhor representado em número de espécies foi Desmidium (5), seguido por Spondylosium (4), Hyalotheca (2), Bambusina, Groenbladia, Onychonema e Teilingia apresentaram apenas uma espécie cada.

\footnotetext{
1 Universidade Estadual de Feira de Santana, Departamento de Ciências Biológicas, Programa de Pós-Graduação em Botânica, Laboratório de Ficologia, Feira de Santana, BA, Brasil

2 Instituto de Botânica, Seção de Ecologia, São Paulo, SP, Brasil

3 Autor para correspondência: ivboliveira@gmail.com
} 


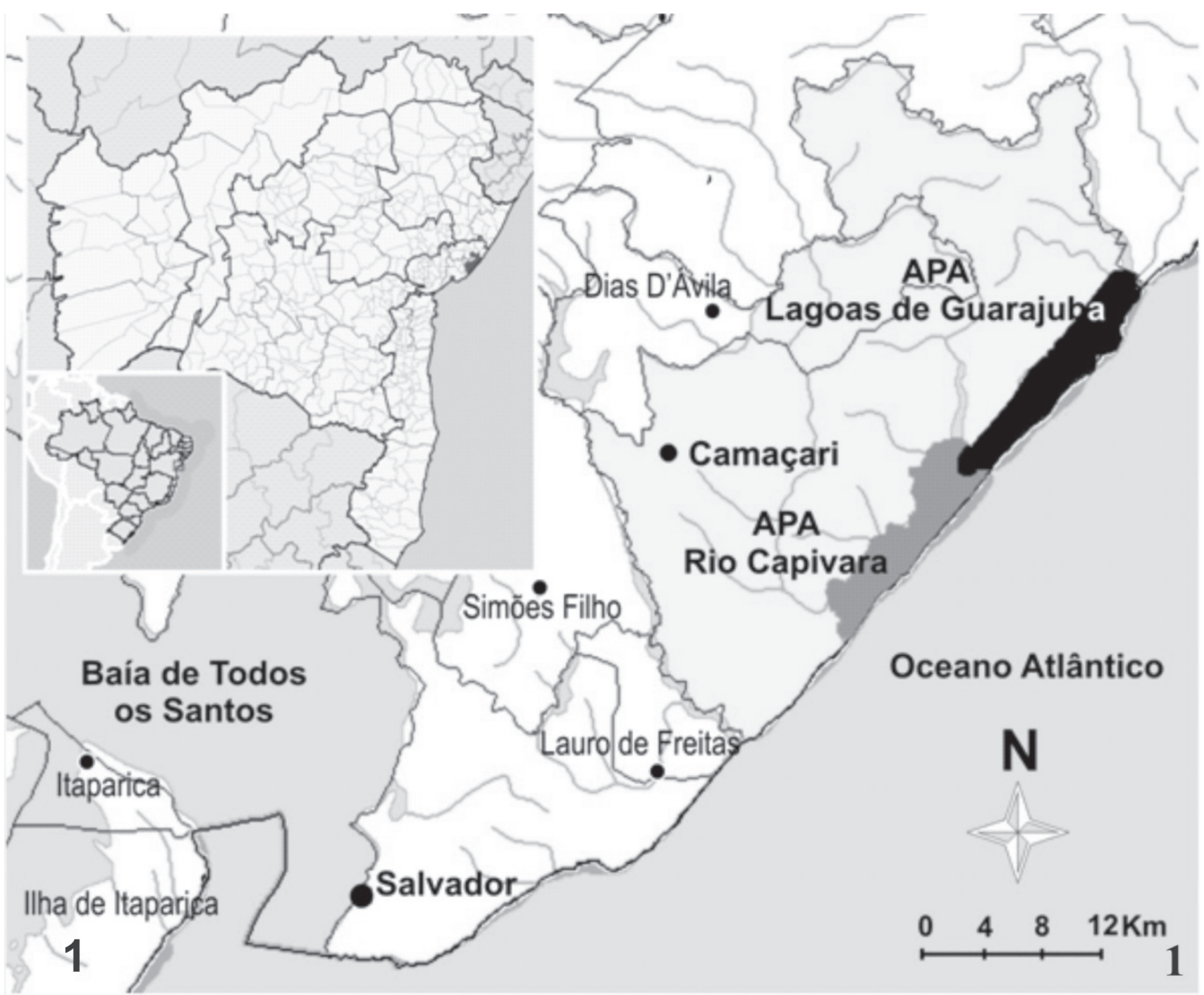

Figura 1. Mapa de localização do estado da Bahia, mostrando em detalhe o município de Camaçari e as duas Áreas de Proteção Ambiental, APA Rio Capivara e APA Lagoas de Guarajuba.

\section{DESMIDIACEAE}

Bambusina Kütz. ex Kütz. 1849, nom. cons.

Bambusina brebissonii Kütz. ex Kütz. var. brebissonii, Spec. Algar. 188. 1849.

Fig. 2-4

Distribuição no Estado da Bahia: Minas-Contas $\left(13,5^{\circ} \mathrm{S}\right.$, $\left.41,5^{\circ} \mathrm{W}\right)$ e Lençóis-Orange $\left(12,5^{\circ} \mathrm{S}, 42^{\circ} \mathrm{W}\right)$ (Förster, 1964 : pl. 35, fig. 3-6, pl. 48, fig. 15). Município de Cairu, Ilha de Tinharé, (Martins \& Bicudo, 1987:fig. 49). Município de Itanagra (Bicudo \& Martins, 1989:fig. 57-58).

Material Examinado: BRASIL. Bahia: Camaçari, APA Lagoas de Guarajuba, 12/I/2007, I.B. Oliveira \& I.S. Oliveira (HUEFS 125504, HUEFS 125508); 08/VI/2007, I.B. Oliveira et al. (HUEFS 125575); 25/VIII/2007, I.B. Oliveira et al. (HUEFS 125611); APA Rio Capivara, 09/III/2007, I.B. Oliveira et al. (HUEFS 125545, HUEFS 125560); 08/
VI/2007, I.B. Oliveira et al. (HUEFS 125571);25/VIII/2007, I.B. Oliveira et al. (HUEFS 125606).

Bicudo \& Samanez (1984), estudando as populações de Bambusina brebissonii var. brebissonii e B. brebissonii var. gracilescens Nordst. no estado de São Paulo, observaram que os limites morfométricos destes se sobrepunham, não sendo possível a distinção entre estas duas variedades. Ainda de acordo com esses autores, o conhecimento de maior número de indivíduos e, principalmente, o estudo de amostras populacionais, fez com que a tênue diferença (dimensões celulares) entre as variedades típica e gracilescens desaparecesse por completo.

Desmidium C.Agardh ex Ralfs 1848

Desmidium aptogonum Bréb. ex Kütz. var. aptogonum, Spec. Algar. 190. 1849.

Fig. 5-6 
Distribuição no Estado da Bahia: Minas-Contas $\left(13,5^{\circ} \mathrm{S}\right.$, 41,5ํ) (Förster, 1964: 435, pl. 36, fig. 1, pl. 51, fig. 5, como Desmidium aptogonum fac. tetragonum). Município de Itanagra (Bicudo \& Martins, 1989: fig. 55-56).

Material Examinado: BRASIL. Bahia: Camaçari, APA Lagoas de Guarajuba, 9/III/2007, I.B. Oliveira et al. (HUEFS 125525), 8/VI/2007, I.B .Oliveira et al. (HUEFS 125575), 20/VII/2007, I.B. Oliveira et al. (HUEFS 125591, HUEFS 125592), APA Rio Capivara, 12/I/2007, I.B. Oliveira \& I.S. Oliveira (HUEFS 125516); 9/III/2007, I.B. Oliveira et al. (HUEFS 125558), 8/VI/2007, I.B. Oliveira et al. (HUEFS 125563, HUEFS 125566, HUEFS 125571), 20/VII/2007, I.B. Oliveira et al. (HUEFS 125583), 25/VIII/2007, I.B. Oliveira et al. (HUEFS 125606).

Borge $(1903,1918,1925)$ e Grönblad (1945) documentaram a ocorrência de Desmidium aptogonum var. aptogonum no Brasil, porém, não ilustraram o material identificado. A primeira referência considerada válida para o Brasil está em Förster (1964), a partir de coletas realizadas em Minas Contas, estado da Bahia. Este autor considerou D. aptogonum como possuindo "facies" tetragona, que se encaixa, perfeitamente, na circunscrição da espécie.

Desmidium baileyi (Ralfs) Nordst. var. baileyi f. baileyi, Acta Univ. Lund. 16: 4. 1880.

Aptogonum baileyi Ralfs, Brit. Desmidieae. 208, pl. 35,

Fig. 1a-C. 1848.

Fig. 7-8

Filamento não ou levemente torcido em hélice; célula tão

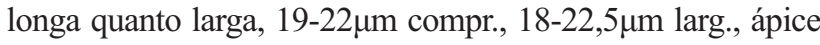
18-22,5 $\mu \mathrm{m}$ larg., istmo 18-22,5 $\mu \mathrm{m}$ larg., contorno quadrangular, muito pouco constrita na parte mediana, seno mediano reduzido a 1 ondulação muito suave; semicélula quadrangular, margens laterais paralelas, às vezes com suaves ondulações, margem superior amplamente côncava, projetada nos ângulos formando 1 processo de conexão cilíndrico, relativamente longo, que adere as células umas às outras por 4 protuberâncias cônicotruncadas, que deixam entre si 1 espaço elíptico-transversal; parede celular hialina, lisa; vista apical 4-angular; cloroplastídio axial, 4-lobulado, cada lóbulo com 1 pirenóide central.

Distribuição no Estado da Bahia: primeira citação de ocorrência da espécie para o Estado.

Material Examinado: BRASIL. Bahia: Camaçari, APA Lagoas de Guarajuba, 12/I/2007, I.B. Oliveira \& I.S. Oliveira (HUEFS 125508), 9/III/2007, I.B. Oliveira et al., (HUEFS 125537), 20/VII/2007, I.B. Oliveira et al., (HUEFS 125591, HUEFS 125592, HUEFS 125596), 25/VIII/2007, I.B. Oliveira et al., (HUEFS 125615, HUEFS 125617), APA Rio Capivara, 12/I/2007, I.B. Oliveira \& I.S. Oliveira (HUEFS 125511, HUEFS 125516), 9/III/2007, I.B. Oliveira et al., (HUEFS 125548), 8/VI/2007, I.B. Oliveira et al., (HUEFS 125563), 20/VII/2007, I.B. Oliveira et al., (HUEFS 125583).

Desmidium baileyi (Ralfs) Nordst f. tetratogonum Nordst. foi proposta por Nordstedt (1888) para identificar espécimes com margens laterais crenadas, com duas ou quatro ondulações. Bicudo \& Samanez (1984) estudaram populações desta espécie e documentaram variação na margem lateral das semicélulas de um mesmo filamento, desde totalmente lisas até crenadas. Desta forma, os autores incluíram $D$. baileyi f. tetratogonum na sinonímia de $D$. baileyi var. baileyi f. baileyi.

Desmidium graciliceps (Nordst.) Lagerh. var. graciliceps, Öfvers. K. svensk VetenskAkad. Förh. 42(7): 228. 1885. Desmidium quadratum Nordst. var. graciliceps Nordst. in Wittr. \& Nordst., Algae Exsic. 8: nº. 367. 1880.

Fig. 9

Distribuição no Estado da Bahia: Minas-Contas (13,5 $5^{\circ}$ $\left.\mathrm{S}, 41,5^{\circ} \mathrm{W}\right)$ e Lençóis Orange $\left(12,5^{\circ} \mathrm{S}, 42^{\circ} \mathrm{W}\right.$ ) (Förster, 1964: 435, pl. 37, fig. 1-3, pl. 51, fig. 1, 10).

Material Examinado: BRASIL. Bahia: Camaçari, APA Lagoas de Guarajuba, 12/I/2007, I.B. Oliveira \& I.S. Oliveira (HUEFS 125504), 9/III/2007, I.B. Oliveira et al., (HUEFS 125525), 20/VII/2007, I.B. Oliveira et al., (HUEFS 125591, HUEFS 125592), APA Rio Capivara 12/I/2007, I.B. Oliveira \& I.S. Oliveira (HUEFS 125511, HUEFS 125521), 9/III/2007, I.B. Oliveira et al., (HUEFS 125545, HUEFS 125548), 20/VII/2007, I.B. Oliveira et al., (HUEFS 125583).

A primeira referência à presença de $D$. graciliceps na Bahia consta em Förster (1964) baseado em coletas realizadas em "Minas Contas" e "Lençóis Orange". A referência apresenta ilustração dos zigósporos, que são típicos da espécie. A espécie é, morfologicamente, próxima de $D$. grevillii de Bary, da qual difere por apresentar células menores e ápices relativamente mais curtos.

Desmidium graciliceps (Nordst.) Lagerh. var. groenbladii C.E.M.Bicudo \& Samanez, Biblioth. Phycol. 68: 39, fig. 11. 1984.

Fig. 10

Filamento levemente torcido em hélice, envolto por abundante bainha de mucilagem, estriada, célula 1,3-1,4 vezes mais longa que larga, 30-32 $\mu \mathrm{m}$ compr., $22-25 \mu \mathrm{m}$ larg., ápice 10-12 $\mu \mathrm{m}$ larg., istmo 18-21 $\mu \mathrm{m}$ larg., contorno elíptico, constrição mediana rasa, seno mediano aberto, linear; semicélula piramidal-truncada, margem apical retusa, margens laterais 2-onduladas, convergentes, ângulos basais acutangular-arredondados; parede celular hialina a levemente acastanhada, lisa; vista apical elíptica, 1 protuberância mamilar em cada pólo; cloroplastídio axial, estelóide, 4-8-lobado; pirenóide 1, central.

Distribuição no Estado da Bahia: primeira citação de ocorrência da espécie para o Estado.

Material Examinado: BRASIL. Bahia: Camaçari, APA Lagoas de Guarajuba, 9/III/2007, I.B. Oliveira et al., (HUEFS 125538), 8/VI/2007, I.B. Oliveira et al., (HUEFS 125575 , HUEFS 125583), APA Rio Capivara, 12/I/2007, I.B. Oliveira \& I.S. Oliveira (HUEFS 125511), 9/III/2007, I.B. Oliveira et al., (HUEFS 125545, HUEFS 125548). 
Os indivíduos representativos desta variedade são bastantes característicos e diferem daqueles da variedade típica da espécie por apresentarem as medidas celulares relativamente maiores e as células 1,3-1,4 vezes mais largas que longas, cujas margens laterais são levemente côncavas na metade superior.

O primeiro registro da ocorrência da variedade está em Grönblad (1945) e foi baseado em material coletado no estado do Pará identificado com a forma "gracilius" sem, entretanto, a pretensão de propor uma novidade taxonômica. Nesse trabalho, o autor não descreveu o material examinado, mas forneceu medidas e uma ilustração. Bicudo \& Samanez (1984) publicaram oficialmente no nível de variedade: var. groenbladii.

Desmidium quadratum Nordst. var. quadratum, Acta Univ. Lund. 9: 49, pl. 1, fig. 24. 1873.

Fig. 11

Filamento torcido em hélice, célula 1,4-1,6 vezes mais larga que longa, 20-22,5 $\mu \mathrm{m}$ compr., 31-33,5 $\mu \mathrm{m}$ larg., ápice

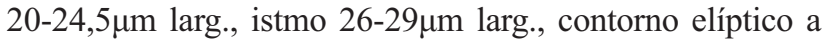
subquadrático, constrição mediana rasa, seno mediano curto, aberto, acutangular ou linear; semicélula piramidal-truncada, ângulos basais arredondados, ângulos apicais retos, margem apical truncada, margens laterais convexas; parede celular hialina a acastanhada, 3 séries de pontuações na região do istmo; vista apical subcircular, 1 proeminência mamilar em cada pólo; cloroplastídio axial, 4-6-lobado; pirenóide 1, central.

Distribuição no Estado da Bahia: primeira citação de ocorrência da espécie para o Estado.

Material Examinado: BRASIL. Bahia: Camaçari, APA Rio Capivara 12/I/2007, I.B. Oliveira \& I.S.Oliveira (HUEFS 125516), 9/III/2007, I.B. Oliveira et al., (HUEFS 125545), 20/VII/2007, I.B. Oliveira et al., (HUEFS 125600 , HUEFS 125606).

Desmidium quadratum var. quadratum é, morfologicamente, próximo de $D$. graciliceps (Nordst.) Lagerh., mas difere por apresentar maior relação entre o comprimento e a largura celulares.

Bicudo \& Samanez (1984) comentaram as referências problemáticas sobre a ocorrência desta espécie no Brasil. Assim, Borge (1918) foi o primeiro a documentar a existência da espécie em território brasileiro ao identificar os exemplares que analisou como "forma major". Grönblad (1945) registrou a ocorrência de duas formas distintas " $m i$ nores" e "maiores" formadas por indivíduos que mediam 13-20 $\mu \mathrm{m}$ compr. x 19-27 $\mu \mathrm{m}$ larg. e ca. $30 \mu \mathrm{m}$ compr. x 33-38 $\mu \mathrm{m}$ larg, respectivamente. Förster (1969) identificou e ilustrou um exemplar como representante de D. quadrum, porém, o material referido não se encaixa na circunscrição dessa espécie.

Groenbladia Teiling 1925

Groenbladia undulata (Nordst.) K.Först. var. undulata, Nova Hedwigia 23(2-3): 578, pl. 26, fig. 10. 1972.
Hyalotheca undulata Nordst. in Wittr. \& Nordst., Algae Exsic. $n^{\circ} 248.1879$.

Fig. 12

Filamento não torcido em hélice, bainha de mucilagem ausente, célula ca. 2,5 vezes mais longa que larga, 18,5-19,5 $\mu \mathrm{m}$ compr., 7,5-8 $\mu \mathrm{m}$ larg., istmo 5-6 $\mu \mathrm{m}$ larg., contorno oblongo, constrição mediana rasa, ampla, seno mediano formado por uma leve invaginação; semicélula aproximadamente cilíndrica, margens laterais aconcavadas, às vezes levemente onduladas, margem apical truncada, ângulos subquadráticos, parede celular hialina, lisa em quase toda extensão, pontuada nos pólos; vista apical circular; vista lateral igual à frontal; cloroplastídios axiais, laminares; pirenóide 1, central.

Distribuição no Estado da Bahia: primeira citação de ocorrência da espécie para o Estado.

Material Examinado: BRASIL. Bahia: Camaçari, Lagoas de Guarajuba, 8/VI/2007, I.B. Oliveira et al., (HUEFS 125575), 20/VII/2007, I.B. Oliveira et al., (HUEFS 125591, 125592), 25/VIII/2007, I.B. Oliveira et al., (HUEFS 125611), APA Rio Capivara 9/III/2007, I.B. Oliveira et al., (HUEFS 125551, HUEFS 125558), 25/VIII/2007, I.B. Oliveira et al., (HUEFS 125606).

De acordo com Bicudo \& Samanez (1984), G. undulata é uma espécie problemática, uma vez que o cloroplastídio de Hyalotheca undulata (basônimo da espécie) é desconhecido e não reconhecível no material tipo. Por outro lado, Bourrelly (1957) e West \& West (1923) o descrevam como sendo típico de Groenbladia.

Hyalotheca Ehrenberg. ex Ralfs 1848

Hyalotheca dissiliens Bréb. ex Ralfs var. tatrica Racib., Pam. Wydz. Akad. Umiejet. 10: 64, pl. 14, fig. 5. 1885. Fig. 13

Filamento simples, não torcido em hélice, envolto por abundante bainha de mucilagem hialina, célula tão longa quanto larga ou 1,3 vezes mais longa que larga, 20-23 $\mu \mathrm{m}$ compr., 16-21 $\mu \mathrm{m}$ larg., istmo 14-19 $\mu \mathrm{m}$ larg., em forma de barrilete, constrição mediana muito leve, porém nítida, seno aberto, raso; semicélula aproximadamente retangular, ângulos arredondados; parede celular hialina, 3 fileiras de pontuações leves, pouco evidentes nas extremidades das células; vista apical cilíndrica; vista lateral igual à frontal; cloroplastídio 1 em cada semicélula, axial, pirenóide 1 , central.

Distribuição no Estado da Bahia: primeira citação da ocorrência da espécie no Estado.

Material Examinado: BRASIL. Bahia: Camaçari, APA Lagoas de Guarajuba, 12/I/2007, I.B. Oliveira \& I.S. Oliveira (HUEFS 125504, HUEFS 125508), 9/III/2007, I.B. Oliveira et al., (HUEFS 125537, HUEFS 125538), 20/VII/2007, I.B.Oliveira et al., (HUEFS 125592), 25/ VIII/2007, I.B. Oliveira et al., (HUEFS 125615), APA Rio Capivara, 12/I/2007, I.B. Oliveira \& I.S. Oliveira (HUEFS 
125511, HUEFS 125516), 9/III/2007, I.B. Oliveira et al., (HUEFS 125545), 8/VI/2007, I.B. Oliveira et al., (HUEFS 125575), 25/VIII/2007, I.B. Oliveira et al., (HUEFS 125605 , HUEFS 125606).

Hyalotheca dissiliens var. tatrica difere da variedade típica da espécie por apresentar células tão longas quanto largas ou até 1,3 vezes mais longas que largas. A identificação dos representantes da variedade é relativamente fácil por conta da forma da célula em barrilete e da constrição mediana bastante leve, mas sempre nítida.

Bicudo \& Samanez (1984) registraram a ocorrência da espécie no Brasil, contudo nas ilustrações que apresentaram não consta bainha de mucilagem. Os exemplares analisados nesse estudo estão de acordo com as medidas apresentadas por estes autores, contudo diferem por apresentar bainha de mucilagem evidente e levemente estriada.

Hyalotheca mucosa (Mertens) Ehrenb. ex Ralfs var. mucosa, Brit. Desmidieae. 53, pl. 1, fig. 2. 1848.

Conferva mucosa Mertens in Dillw., Brit. Confervea. 46, pl. B, 1809.

Fig. 14

Filamento longo, imerso em abundante bainha de mucilagem, hialina, célula pequena, 1,1-1,3 vezes mais longas

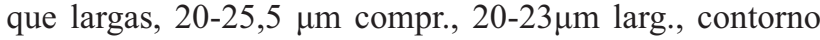
quadrático a retangular, sem a menor indicação da constrição mediana, semicélula transversalmente retangular, margens laterais lisas, paralelas entre si, levemente convergentes no sentido do ápice, margem apical truncada a levemente arredondada; parede celular hialina, 3 anéis de grânulos localizados no ápice da semicélula, demais regiões lisas; vista lateral igual à frontal; cloroplastídio axial, irregular; pirenóide 1, central.

Distribuição no Estado da Bahia: Município de Itanagra (Bicudo \& Martins, 1989: fig. 59).

Material Examinado: BRASIL. Bahia: Camaçari, APA Lagoas de Guarajuba, 9/III/2007, I.B. Oliveira et al., (HUEFS 125540), 20/VII/207, I.B. Oliveira et al., (HUEFS 1125591, HUEFS 125596), 25/VIII/2007, I.B. Oliveira et al., (HUEFS 125611, HUEFS 125613, HUEFS 125615), APA Rio Capivara, 12/I/2007, I.B. Oliveira \& I.S. Oliveira (HUEFS 125511), 9/III/2007, I.B. Oliveira et al., (HUEFS 125545, HUEFS 125560), 8/VI/2007, I.B. Oliveira et al., (HUEFS 125563, HUEFS 125566, HUEFS 125571), 25/VIII/2007, I.B. Oliveira et al., (HUEFS 125605, HUEFS 125606).

Hyalotheca mucosa var. mucosa é uma espécie bem delimitada na literatura, apresentando célula de contorno quadrático a retangular, sem a menor indicação de constricção mediana.

De acordo com Bicudo \& Samanez (1984), H. mucosa (Mertens) Ehrenb. ex Ralfs pode apresentar células tão longas quanto largas, até quase duas vezes mais longas ou até 1,5 vezes mais largas.
Onychonema G.C.Wall. 1860

Onychonema laeve (Nordst.) var. latum West \& G.S.West, Trans. Linn. Soc. Lond.: sér.2, 5(5):232, pl. 12, fig. 18. 1896.

Fig. 15-16

Filamento não ou levemente torcido em hélice, 1,21,4 vezes mais longa que larga, sem incluir os espinhos, 22,5-25 $\mu \mathrm{m}$ compr. incl. espinhos, 15-17,5 $\mu \mathrm{m}$ compr. excl. espinhos, 26-28,5 $\mu \mathrm{m}$ larg. incl. espinhos, 21-22,5 $\mu \mathrm{m}$ larg. excl. espinhos, istmo 2,5-5 $\mu \mathrm{m}$ larg., contorno subretangular, constrição mediana profunda, seno mediano inicialmente linear, fechado, a seguir aberto, semicélulas oblongas, margem basal amplamente arredondada, ângulo basal com 1 espinho robusto, curvo, pontiagudo, convergente, margem superior amplamente côncava, 2 processos curtos diagonalmente simétricos que se sobrepõem aos da célula vizinha; parede celular hialina, lisa, às vezes finamente pontuada; vista apical não observada; vista lateral oval, 1 espinho pontiagudo em cada polo; cloroplastídio axial; pirenóide 1, central.

Distribuição no Estado da Bahia: primeira citação de ocorrência da espécie para o Estado.

Material Examinado: BRASIL. Bahia: Camaçari, APA Lagoas de Guarajuba, 8/VI/2007, I.B. Oliveira et al., (HUEFS 125575), APA Rio Capivara, 9/III/2007, I.B. Oliveira et al., (HUEFS 12555), 20/VII/2007, I.B. Oliveira et al., (HUEFS 125600).

Durante este estudo, observamos células 1,2-1,4 vezes mais longas que largas, que diferem daquelas relatadas por Bicudo \& Samanez (1984) e daquelas da descrição original de West \& West (1896), onde os autores documentaram células cerca de duas vezes mais longas que largas. Contudo, a presença de características tais como: (1) margem superior reta; (2) curta distância no meio, depois pronunciadamente arqueada até o ângulo espinífero; e (3) vista vertical estreita, com lados quase retos, permitiram identificar o material estudado com $O$. laeve var. latum:

Spondylosium Bréb. ex Kütz. 1849

Spondylosium desmidiiforme (Borge) West, Journ. Bot. 42: 286, pl. 464, fig. 11-12 (13?). 1904.

Sphaerozosma desmidiiforme Borge, Ark. Bot. 1: 120, pl. 5, fig, 23a-C. 1903.

Fig. 17

Distribuição no Estado da Bahia: Minas-Contas $\left(13,5^{\circ} \mathrm{S}\right.$, $41,5^{\circ} \mathrm{W}$ ) e Lençóis-Orange $\left(12,5^{\circ} \mathrm{S}, 42^{\circ} \mathrm{W}\right)$ (Förster, 1964 : pl. 35, fig. 9-11, pl. 44, fig. 11, pl. 51, fig. 2-3, 8). Município de Cairu, Ilha de Tinharé, (Martins \& Bicudo, 1987: fig. 49). Município de Itanagra, (Bicudo \& Martins, 1989: fig. 54).

Material Examinado: BRASIL. Bahia: Camaçari, APA Lagoas de Guarajuba, 9/III/2007, I.B. Oliveira et al., (HUEFS 125530, HUEFS 125531, HUEFS 125540), APA Rio Capivara, 12/I/2007, I.B. Oliveira \& I.S. Oliveira (HUEFS 125516, HUEFS 125521), 9/III/2007, I.B. Oliveira et al., (HUEFS 125545, HUEFS 125555, HUEFS 125558). 

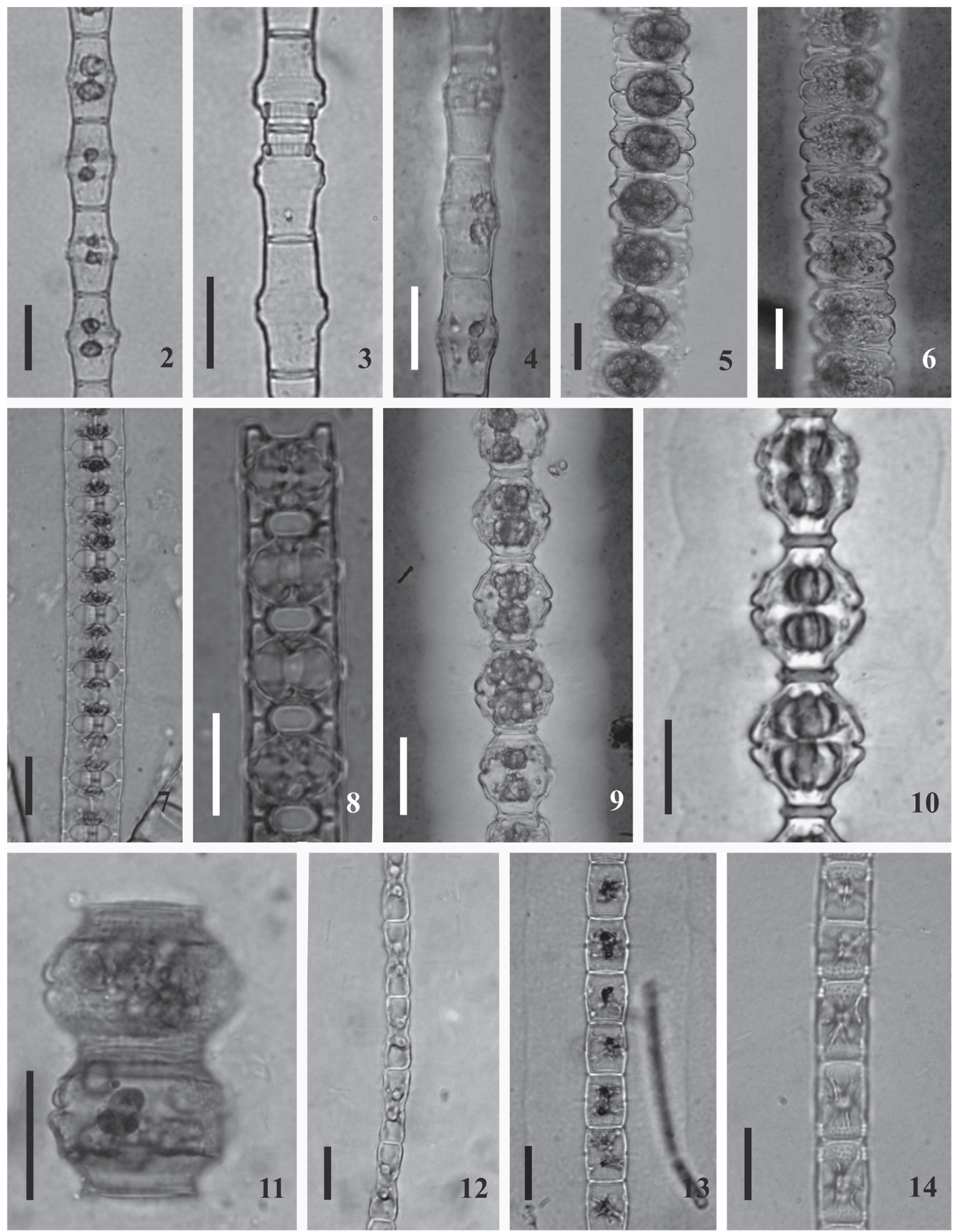

Figuras 2-14. Desmídias filamentosas das APAs Lagoas de Guarajuba e Rio Capivara. 2-4. Bambusina brebissonii Kütz. ex Kütz. var. brebissonii; .5-6. Desmidium aptogonum Bréb. ex Kütz. var. aptogonum; 7-8. Desmidium baileyi (Ralfs) Nordst. var. baileyi f. baileyi; 9. Desmidium graciliceps (Nordst.) Lagerh. var. graciliceps; 10. Desmidium graciliceps (Nordst.) Lagerh. var. groenbladii C.E.M.Bicudo \& Samanez; 11. Desmidium quadratum Nordst. var. quadratum; 12. Groenbladia undulata (Nordst.) K.Först. var. undulata;. 13. Hyalotheca dissiliens Bréb. ex Ralfs var. tatrica Racib.; 14. Hyalotheca mucosa (Mertens) Ehrenb. ex Ralfs var. mucosa. Escala $=20 \mu \mathrm{m}$. 
Förster (1964), pioneiramente, documentou a espécie para o estado da Bahia, descrevendo a variação da forma das semicélulas, medidas celulares e a abertura do seno mediano.

De acordo com as variações apresentadas por Förster (1964), Bicudo \& Samanez (1984) resolveram considerar sinônimos taxonômicos (heterotípicos) de S. desmidiiforme os nomes $S$. desmidiiforme var. desmidiiforme f. tenuius Först. e $S$. desmidiiforme var. desmidiiforme f. desmidiiforme, em virtude de não haverem conseguido estabelecer, na amostra populacional estudada, os limites inicialmente propostos para as duas formas.

Spondylosium panduriforme (Heimerl) Teiling var. panduriforme f. limnetica (West \& G.S.West) Teiling, Svenska Bot. Tidsskr. 51(1): 216, pl. 210, fig, 12-14. 1957. Fig. 18-19

Filamentos simples, sem bainha de mucilagem evidente, $1,6-1,7$ vezes mais longa que larga, $30-31 \mu \mathrm{m}$ compr., 17,5-18,5 $\mu \mathrm{m}$ larg., istmo 10-11 $\mu \mathrm{m}$ larg., contorno oblongo, constrição mediana mais ou menos profunda, ampla, seno mediano aberto; semicélula subcircular, às vezes subelíptica, células unidas umas às outras pela região mediana da margem apical que, muitas vezes é uma leve acuminação, margens laterais côncavas, margem apical amplamente arredondada, levemente acuminada, ângulos arredondados; parede celular hialina, lisa; vista apical elíptica; cloroplastídio pseudoestelóide; pirenóide 1, central.

Distribuição no Estado da Bahia: primeira citação de ocorrência da espécie para o Estado.

Material Examinado: BRASIL. Bahia: Camaçari, APA Lagoas de Guarajuba, 12/I/2007, I.B. Oliveira \& I.S. Oliveira (HUEFS 125504, HUEFS 125508), 9/III/2007, I.B. Oliveira et al., (HUEFS 125538), 20/VII/2007, I.B. Oliveira et al., (HUEFS 125592, HUEFS 125596), 25/VIII/2007, I.B. Oliveira et al., (HUEFS 125611, HUEFS 125613), APA Rio Capivara, 12/I/2007, I.B. Oliveira \& I.S. Oliveira (HUEFS 125516), 9/III/2007, I.B. Oliveira et al., (HUEFS 125545, HUEFS 125560, HUEFS 125563, HUEFS 125566, HUEFS 125571, HUEFS 125573), 20/VII/2007, I.B. Oliveira et al., (HUEFS 125600), 25/VIII/2007, I.B. Oliveira et al., (HUEFS 125606).

A atual f. limnetica difere da típica da espécie por apresentar as medidas lineares das células superiores as da forma típica.

Croasdale et al. (1983) comentaram a semelhança morfológica existente entre $S$. panduriforme var. panduriforme $\mathrm{f}$. limnetica e Cosmarium moniliforme (Turpin) Ralfs. Apesar destes táxons apresentem formas e dimensões celulares similares, C. moniliforme difere por apresentar a vista apical da célula circular e o cloroplastídio lobado.

Spondylosium planum (Wolle) West \& G.S.West, Journ. Linn. Soc. Lond.: sér. Bot. 40: 430, pl. 19, fig. 5-8. 1912.
Sphaerozosma pulchrum Bailey var. planum Wolle, Desm.U.S. 20, pl. 4, fig. 3-4. 1884.

Fig. 20-21

Filamento não torcido em hélice, bainha de mucilagem não evidente, célula 1,1-1,2 vezes mais larga que longa, 8-11 $\mu \mathrm{m}$ compr., 9-12 $\mu \mathrm{m}$ larg., istmo 4-6 $\mu \mathrm{m}$ larg., aderidas umas às outras em toda superfície polar, contorno subquadrático, constrição mediana profunda, seno mediano aberto, obtusângulo; semicélula transversalmente elíptica a oblonga, margens laterais amplamente arredondadas, margem superior plana ou levemente convexa; parede celular hialina, lisa; vista apical oblonga; cloroplastídio axial, lobulado; pirenóide central.

Distribuição no Estado da Bahia: primeira citação de ocorrência da espécie para o Estado.

Material Examinado: BRASIL. Bahia: Camaçari, APA Lagoas de Guarajuba, 12/I/2007, I.B. Oliveira \& I.S. Oliveira (HUEFS 125504), 9/III/2007, I.B. Oliveira et al., (HUEFS 125537, HUEFS 125540), 20/VII/2007, I.B. Oliveira et al., (HUEFS 125592), APA Rio Capivara, 9/III/2007, I.B. Oliveira et al., (HUEFS 125545, HUEFS 125548, HUEFS 125555), 8/VI/2007, I.B. Oliveira et al., (HUEFS 125566).

O primeiro registro de ocorrência de Spondylosium planum no território brasileiro está em Grönblad (1945), ao listar este para o estado do Pará. Posteriormente, Förster (1969) apresenta descrição, medidas e ilustrações para espécimes também coletados no Pará.

Spondylosium pulchrum (Bailey) W.Archer var. pulchrum, In Pritchard, Infusoria. 724. 1861.

Sphaerozosma pulchrum Bailey, in Ralfs, Brit. Desmidieae. 209, pl. 35, fig. 2. 1848.

Fig. 22-23

Distribuição no Estado da Bahia: Município de Itanagra (Bicudo \& Martins, 1989: fig. 60).

Material Examinado: BRASIL. Bahia: Camaçari, APA Lagoas de Guarajuba, 12/I/2007, I.B. Oliveira \& I.S. Oliveira (HUEFS 125508), 9/III/2007, I.B. Oliveira et al., (HUEFS 125531, HUEFS 125540), 20/VII/2007, I.B. Oliveira et al., (HUEFS 125600), APA Rio Capivara, 8/VI/2007, I.B. Oliveira et al., (HUEFS 125563), 25/VIII/2007, I.B. Oliveira et al., (HUEFS 125606).

$\mathrm{Na}$ literatura especializada, Spondylosium pulchrum var. brasiliense Nordst. e Spondylosium pulchrum var. pulchrum, tem sido separados apenas quanto a vista apical das semicélulas, que na primeira é oblonga, com uma constrição bem evidente no meio e na segunda, é elíptica.

Segundo Bicudo \& Samanez (1984), que contém profusa documentação sobre Spondylosium pulchrum, essas características são extremamente variáveis até num mesmo filamento, o que os levou a considerar as duas variedades taxonomicamente idênticas e do ponto de vista nomenclatural sinônimos.

Leite \& Senna (1992) relataram a presença de um glóbulo de mucilagem acastanhada entre as projeções, unindo as 

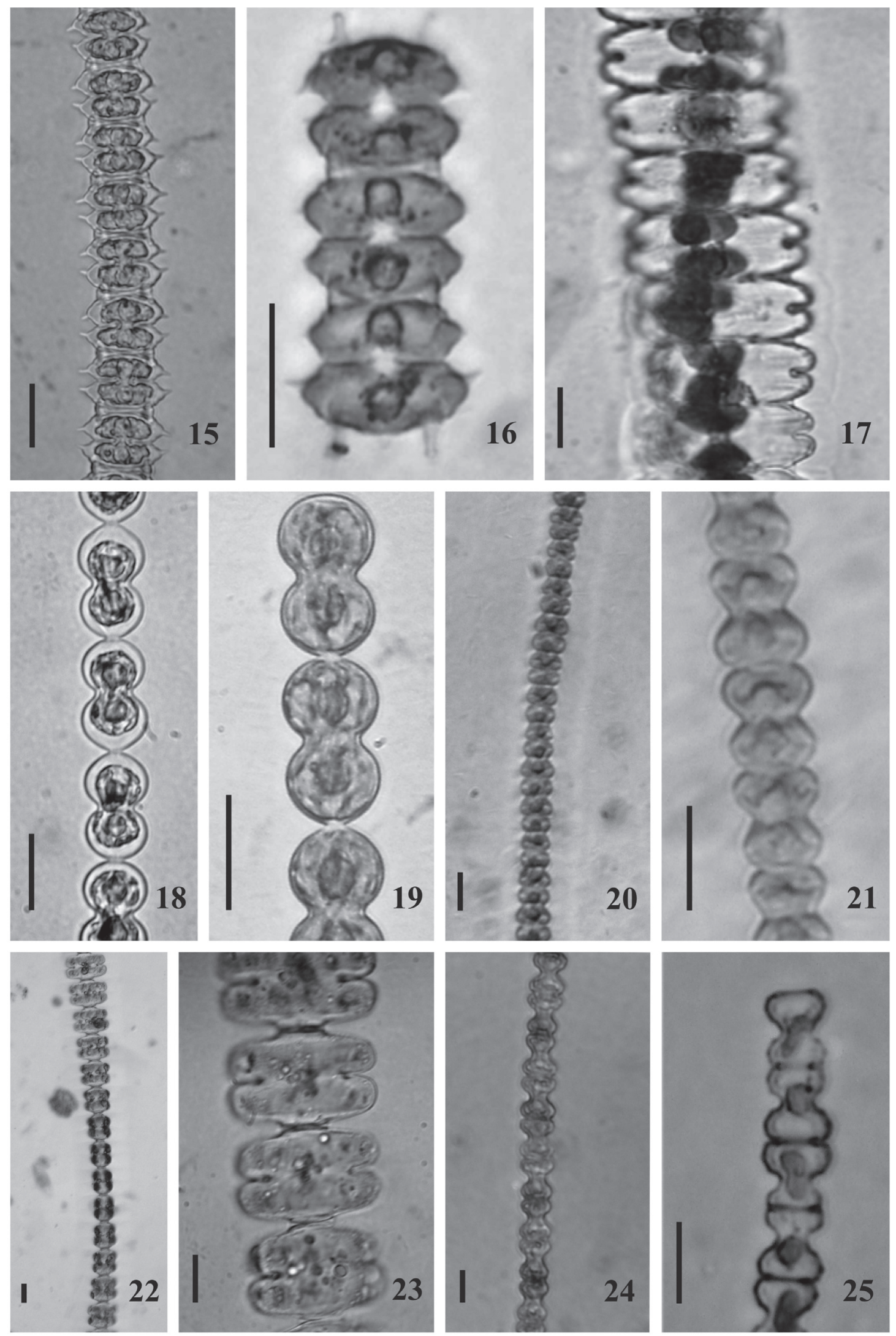

Figuras 15-25. Desmídias filamentosas das APAs Lagoas de Guarajuba e Rio Capivara. 15-16. Onychonema laeve (Nordst.) var. latum West \& G.S.West; 17. Spondylosium desmidiiforme (Borge) West; 18-19. Spondylosium panduriforme (Heimerl) Teiling var. panduriforme f. limnetica (West \& G.S. West) Teiling; 2021. Spondylosium planum (Wolle) West \& G.S.West; 22-23. Spondylosium pulchrum (Bailey) W.Archer var. pulchrum; 24-25. Teilingia granulata (Roy \& Bisset) Bourr. Escala $=20 \mu \mathrm{m}$ 
células umas às outras no filamento. Tal glóbulo jamais foi observado nas populações presentemente estudadas.

\section{Teilingia Bourr. 1964}

Teilingia granulata (Roy \& Bisset) Bourr., Revue Algologique: sér. 2, 7(2): 190. 1964.

Sphaerozosma granulatum Roy \& Bisset, Journ. Bot. 24: 242, pl. 268, fig. 17. 1886.

Fig. 24-25

Filamento simples, raramente torcido em hélice, bainha mucilaginosa às vezes presente, células tão longas quanto largas, 8-10 $\mu \mathrm{m}$ compr., 8,5-10 $\mu \mathrm{m}$ larg., istmo 3,5-4,5 $\mu \mathrm{m}$ larg., contorno subquadrático, constrição mediana profunda, seno mediano aberto, semicircular; semicélula transversalmente oblonga, margens laterais amplamente arredondadas; 3 grânulos em cada margem, 1-2 intramarginais, margem apical reta, 1 grânulo em cada ângulo; vista apical da célula oblonga, 3 grânulos em cada pólo; parede celular hialina, lisa; cloroplastídio axial; pirenóide 1, central.

Distribuição no Estado da Bahia: primeira citação de ocorrência da espécie para o Estado.

Material Examinado: BRASIL. Bahia: Camaçari, APA Lagoas de Guarajuba, 8/VI/2007, I.B.Oliveira et al., (HUEFS 125575), 20/VII/2007, I.B.Oliveira et al., (HUEFS 125592, HUEFS 125596), 25/VIII/2007, I.B.Oliveira et al., (HUEFS 126511, HUEFS 125615), APA Rio Capivara 20/ VII/2007, I.B.Oliveira et al., (HUEFS 125600).

Förster (1969) documentou a ocorrência da espécie na Amazônia, como Sphaerozosma granulatum Roy \& Bisset, porém, não consta nesse trabalho descrição nem qualquer comentário sobre o material estudado, apenas as medidas celulares $(7,5-11 \mu \mathrm{m} \times 7,5-9 \mu \mathrm{m})$ e ilustrações, as quais estão de acordo com o material analisado neste estudo.

O trabalho de Bicudo \& Bicudo (1965) é o primeiro a trazer o registro da espécie como $S$. granulatum Roy \& Bisset, com descrição e ilustração do material, a partir de coletas realizadas em tanques artificiais, valeta e empoçados no Instituto de Botânica de São Paulo.

Teilingia granulata morfologicamente, é semelhante a T. wallichii (Roy \& Bisset) Bourr. var. anglica (West \& G.S.West) K.Först., mas difere por apresentar seno mediano em forma de "U" e um conjunto de até oito grânulos na face de cada semicélula.

Foram identificados 15 táxons de desmídias filamentosas, dos quais apenas Bambusina brebissonii var. brebissonii, Desmidium aptogonum var. aptogonum, D. graciliceps var. graciliceps, Hyalotheca mucosa, Spondylosium desmidiiforme e S. pulchum já haviam sido registrados em trabalhos anteriores para o estado da Bahia.

Os nove táxons seguintes são adições à desmidioflórula da Bahia: Desmidium baileyi var. baileyi f. baileyi, D. graciliceps var. groenbladii, D. quadratum var. quadratum,
Groenbladia undulata var. undulata, Hyalotheca dissiliens var. tatrica, Onychonema laeve var. latum, Spondylosium panduriforme var. panduriforme f. limnetica, S. planum, Teilingia granulata, aumentando o total de espécies conhecidas para 22.

Spondylosium panduriforme var. panduriforme f. limnetica e Hyalotheca mucosa var. mucosa foram as espécies de ocorrência mais freqüente durante o atual estudo, por estarem presentes em $50 \%$ e $44,8 \%$, respectivamente, das amostras analisadas. Ao passo que Desmidium quadratum var. quadratum e Onychonema laeve var. latum foram as espécies de ocorrência menos freqüente durante o atual estudo, com $12,8 \%$ e $9,6 \%$, respectivamente, nas amostras analisadas.

Dentre os quinze táxons inventariados, apenas Desmidium quadratum var. quadratum foi exclusivo da APA Lagoas de Guarajuba.

Com relação aos períodos amostrados, verificou-se que Spondylosium desmidiiforme foi registrado apenas nos meses de verão ao passo que Teilingia granulata foi registrada apenas no período de inverno.

\section{Agradecimentos}

À Coordenação de Aperfeiçoamento de Pessoal do Ensino Superior, pela concessão da Bolsa de Mestrado à primeira autora; à Universidade Estadual de Feira de Santana (UEFS) e ao Instituto de Botânica da Secretaria do Meio Ambiente do Estado de São Paulo, pelo apoio logístico; ao Programa de Pós-Graduação em Botânica da UEFS, pelo auxílio financeiro concedido para a realização das coletas.

\section{Referências bibliográficas}

Bicudo, C.E.M. 1969. Contribution to the knowledge of the state of São Paulo, Brazil (including from the state of Minas Gerais). Nova Hedwigia 17: 443-549.

Bicudo, C.E.M. \& Bicudo, R.M.T. 1965. Contribuição ao conhecimento das Desmidiaceae do Parque do Estado, São Paulo. 2. Rickia 2: 39-54.

Bicudo, C.E.M. \& Samanez, I.M. 1984. Desmidioflórula Paulista III. Bibliotheca Phycologica 68: 1-139.

Bicudo, C.E.M. \& Martins, D.V. 1989. Desmídias (Zygnemaphyceae) de Itanagra, estado da Bahia, Brasil. Revista Brasileira de Biologia 49: 309-324.

Bicudo, C.E.M.; Sormus, L. \& Schetty, S.P. 1999. Criptógamos do Parque Estadual das Fontes do Ipiranga, São Paulo, SP. Algas, 12: Zygnemaphyceae (Desmidiaceae, 2: Desmidium, Groenbladia, Hyalotheca, Spondilosium e Teilingia). Hoehnea 26: 75-85.

Bicudo, C.E.M. \& Menezes, M. 2006. Gêneros de algas de águas continentais do Brasil: chave para identificação e descrições. São Carlos, RiMa.

Borge, O. 1903. Die Algen der ersten Regnellschen Expedition, 2: Desmidiaceae. Arkiv för Botanik 1: 71-138.

Borge, O. 1918. Die von Dr. A. Löfgren in São Paulo gessammelten Süsswasseralgen. Arkiv för Botanik 15: 1-108.

Borge, O. 1925. Die von F.C. Hoehne Wahrend der espedition Roosevelt-Rondon gessammelten Süsswasseralgen. Arkiv för Botanik 19: 1-56.

Bourrelly, P. 1957. Recherches sur les Chrysophycdes. Morphologie, phylogenie, systematique. Ph.D. thesis. La Faculty des Sciences de L'University de Paris.

Brook, A.J. 1981. The biology of desmids. London, Blackwell Scientific Publishers.

Brook, A.J. \& John, D.M. 2002. Phyllum Chlorophyta (Green Algae) Ordem Zygnematales. Pp. 479-593. In: John, D.M.; Whiton, B.A. \& 
Brook, A.J. (eds). The freshwater algal flora of the British Isles: an identification guide of freshwater and terrestrial algae. Cambridge, Cambridge University Press.

Croasdale, H.T.; Bicudo, C.E.M. \& Prescott, G.W. 1983. A synopsis of North American Desmids. Part II. Desmidiaceae, Placodermae. Section 5. Lincon, University of Nebraska Press.

Coesel, P.F.M. 1994. De Desmidiaceeën van Neerland. Fam. Desmidiaceae. v. 5. Utrecht, Stichting Uitgeverj Koninklijke Nederlandse Natuurhistorische Vereniging.

De-Lamonica-Freire, E.M. 1992. Desmídias filamentosas (Zygnemaphyceae, Desmidiales) da estação ecológica da Ilha de Taimã, Mato Grosso, Brasil. Acta Limnológica Brasileira IV: 315-325.

Delazari-Barroso, A.; Sant'anna, C.L. \& Senna, P.A.C. 2007. Phytoplankton from Duas Bocas Reservoir, Espírito Santo State, Brazil (except diatoms). Hoehnea 34: 211-229.

Dias, I.C.A. \& Sophia, M.G. 1994. Flora dos estados de Goiás e Tocantins, Criptógamos: Desmidiaceae, Odogoniaceae, Zygnemaceae. Goiânia, Cegraf/UFG 3.

Förster, K. 1964. Desmidiaceen aus Brasilien. 2: Bahia, Goyaz, Piauhy und Nord-Brasilien. Hydrobiologia 23: 321-505.

Förster, K. 1969. Amazonische desmidien, 1. Amazoniana 2: 5-116.

Förster, K. 1974. Amazonische desmidien, 2. Amazoniana 2: 135-242.
Grönblad, R. 1945. De algus brasiliensibus: praecipue Desmidiacius, in regione inferiore fluminis Amazonas, Acta Societatis Scientiarum Fennicae, nov., ser. B, 2(6): 1- 43

Ling, H.U. \& Tyler, P.A. 1980. Freshwater algae of the Alligator rivers, region northern territory of Australia. Tasmania, University of Tasmania.

Leite, A.L.T.A. \& Senna, P.A.C. 1992. Desmídias (Zygnemaphyceae) da Lagoa Bonita, Distrito Federal, Brasil, 1: gêneros filamentosos. Hoehnea 19: 93-104.

Martins, D.V. \& Bicudo, C.E.M. 1987. Desmídias da Ilha de Itanharé, estado da Bahia, Brasil. Revista Brasileira de Biologia 47: 1-16.

Nordstedt, O. 1879. Algologiska småsaker II. Vaucheria studier, 1879. Botaniska Notiser 1879: 177-90.

Nordstedt, O. 1888. Fresh-water algae, collected by Dr. S. Berggren in New Zeland and Australia. Kong. Kungliga Svenska Vetenskapsakademiens Handlingar 22: 1-98.

Scott, A.M; Grönblad, R. \& Croasdale, H. 1965. Desmids from the Amazon Basin, Brasil. Acta Botanica Fennica 69: 1-93.

West, W. \& West, G.S. 1896. On some North American desmidieae. Journal of the Linnean Society. Botany 5: 229-279.

West, W.; West, G. S.; Carter, N. 1923: A monograph of the British Desmidiaceae. The Ray Society 5: 129-167.

Versão eletrônica do artigo em www.scielo.br/abb e http://www.botanica.org.br/acta/ojs 